\title{
Evaluation of Placental Blood Flow in Patients with Placental Insufficiency
}

\author{
Julia E. Dobrokhotova, PhD, $\mathrm{ScD}^{1 *}$; Sophia A. Zalesskaya ${ }^{1}$; Elena A. Zubareva, PhD, \\ $\mathrm{ScD}^{1}$; Andrey R. Zubarev, $\mathrm{PhD}, \mathrm{ScD}^{1}$; Tatiana A. Rumyantseva, $\mathrm{PhD}^{1,2}$ \\ ${ }^{I}$ N.I. Pirogov Russian National Research Medical University \\ ${ }^{2}$ Central Research Institute of Epidemiology \\ Moscow, the Russian Federation
}

\begin{abstract}
Background: Placental insufficiency is a major problem of modern obstetrics due to its link to maternal and perinatal morbidity and mortality. Placental microcirculatory disorders play a decisive role in the pathogenesis of this condition. Thus, an evaluation of placental blood flow is of particular importance and crucial for appropriate diagnosis. The aim of this study was to evaluate placental blood flow in patients with placental insufficiency. SMI (superb microvascular imaging) was compared to color Doppler for that purpose.

Materials and Methods: Primigravida patients $(\mathrm{n}=91)$ at 15 to 16 weeks of gestation were enrolled. Inclusion criteria were spontaneous singleton pregnancy, age from 18 to 45 years. All participants were divided into 2 groups: Group 1 - control group $(\mathrm{n}=27)$ and Group 2 - threatened miscarriage group $(\mathrm{n}=64)$. Transvaginal ultrasound and color Doppler were performed to assess uteroplacental circulation. Placental blood flow was evaluated using a Toshiba Aplio ${ }^{\text {TM }} 500$ machine equipped with an SMI tool.

Results: Placental blood flow assessment in patients with normal pregnancy revealed homogenous placental tissue, normal distribution of vessels, and active blood flow; in patients with pregnancy complications, we found inhomogeneous placenta, decreased blood flow, sporadic vessels, and avascular areas. SMI demonstrated several benefits compared to color Doppler imaging. Color Doppler allows us to assess superficial vessels only, whereas SMI provides more comprehensive data on the overall vascularization of the placenta.

Conclusion: SMI by Aplio ${ }^{\mathrm{TM}} 500$ (Toshiba) may be an effective tool in the assessment of placental blood flow and the diagnosis and prognosis of placental insufficiency. (Int J Biomed. 2017; 7(1):28-31.)
\end{abstract}

Key Words: superb microvascular imaging $\bullet$ placental circulation $\bullet$ threatened miscarriage $\bullet$ placental insufficiency

\section{Introduction}

Spontaneous abortion in early pregnancy remains an urgent problem in modern obstetrical care..$^{[1]}$ According to previous studies, spontaneous miscarriage occurs during the first trimester of pregnancy in $50 \%$ cases, during the second trimester of pregnancy - in $20 \%$ cases, and during the third trimester of pregnancy - up to $30 \%$ cases. ${ }^{[2]}$ Uterus hyper tone is especially unfavorable in the first trimester of pregnancy when trophoblast invasion and placental formation are initiated.

*Corresponding author: Professor Julia E. Dobrokhotova, PhD, ScD. Head of Department of Obstetrics and Gynecology, Medical Faculty, N.I. Pirogov Russian National Research Medical University. Moscow, the Russian Federation.E-mail: pr.dobrohotova@mail.ru
Deterioration of those processes may influence the further course of pregnancy. Spiral arteries are of major importance in the course of placental development. Spiral arteries are the terminal parts of uterine vessels, which penetrate the implantation and placentation area. Conversion of spiral arteries implies 5-10 fold dilation at the mouth of the vessels, which are temporarily blocked by extravillous trophoblast. By the 12th week of gestation, these vessels are unblocked, thus initiating maternal blood flow in the intervillous space. The absence of trophoblastic plugs along with deterioration of trophoblast invasion leads to pathological placentation. ${ }^{[3,4]}$ Pathological placentation leads to the formation of preterm maternal blood flow and the increase in oxygen concentration in the intervillous space. Those problems in early pregnancy may affect chorionic villi, leading to retroplacental or subchorionic 
hematomas and spontaneous abortion. ${ }^{[4,5]}$ Placental insufficiency is a major problem of modern obstetrics due to its link to maternal and perinatal morbidity and mortality. ${ }^{[1,2]}$ Placental insufficiency is classified according to the period of its onset (primary placental insufficiency, arising before 16 weeks of gestation, and secondary, arising after 16 weeks of gestation). Violations of the implantation process (defective trophoblast invasion and disruption of placental angiogenesis activity) cause pregnancy to take an abnormal course. ${ }^{[6,7]}$ Microcirculatory disorders in the intervillous space play the major role in the pathogenesis of placental insufficiency, eventually leading to circulatory disorders in the motherplacenta-fetus system and to intrauterine fetal hypoxia and fetal retardation. ${ }^{[8]}$ According to some studies, intraplacental circulatory disorders are accompanied by circulatory disorders in certain areas of the placenta. ${ }^{[9]}$ Thus, an evaluation of placental blood flow is of particular importance and crucial for appropriate diagnosis. Ultrasound is one of the methods that offer a great perspective in the field. Color and pulsed Doppler as well as 3D reconstruction of vessels in the placenta are widely used in obstetrics and gynecology, while placental microcirculation evaluation is still under study. ${ }^{[10,11]}$ The ultrasound SMI (superb microvascular imaging) technique has been recently introduced. SMI allows visualization of the smallest vessels characterized by low-velocity flows. Moreover, SMI implies high-definition techniques, which minimize the risk of registration of artifacts. Considering the benefits of the method, we tried to apply it to the visualization of utero-placental blood flow as a diagnostic tool for placental insufficiency.

The aim of this study was to evaluate placental blood flow in patients with placental insufficiency. SMI was compared to color Doppler for that purpose.

\section{Materials and Methods}

Primigravida patients $(n=91)$ at 15 to 16 weeks of gestation were enrolled. Inclusion criteria were spontaneous singleton pregnancy, age from 18 to 45 years. Exclusion criteria were vaginal infections, congenital anomalies of the genitalia, ovarian and adrenal hyperandrogenism, thyroid gland diseases, other extragenital diseases, multiple gestation, and pregnancy as a result of assisted reproductive treatment. Last normal menstrual period was used to calculate the gestational age.

All participants were divided into 2 groups: Group 1 - control group $(n=7)$ and Group 2 - threatened miscarriage group $(n=64)$. Informed consent was signed by each participant. Threatened miscarriage was diagnosed by ultrasound. Pain and discomfort in the lower abdominal region were the leading complains (75\%) in Group 2 upon admission. Vaginal bleeding was registered in $25 \%$ patients in Group 2. Myometrial hypertonus (78\%), chorion abruption accompanied by retrochorial hematoma (12\%) and cervical shortening $(17 \%)$ were identified by ultrasound. Clinical data were collected and pelvic exam performed for all patients. The two groups did not vary significantly in terms of age, extragenital diseases, or sociodemographical characteristics.
Transvaginal ultrasound and color Doppler were performed to assess uteroplacental circulation. Placental blood flow was evaluated using a Toshiba Aplio ${ }^{\text {TM }} 500$ machine equipped with an SMI tool. Vascularization index (VI) was automatically calculated in all cases.

Examination and analysis included the following steps:

1. Visualization of placental vascularization by SMI.

Several parameters were evaluated:

a) uniformity of vascularization,

b) intensity of vascularization

c) avascular areas.

2. Visual assessment of intensity of vascularization in a certain area.

3. Evaluation of VI

4. Comparison of SMI and color Doppler.

The statistical analysis was performed using the statistical software «Statistica». (v6.0, StatSoft, USA). For data with normal distribution, inter-group comparisons were performed using Student's t-test. A probability value of $\mathrm{P}<0.05$ was considered statistically significant.

\section{Results}

Mean age of participants was $23.9 \pm 3.5$ years. Placental blood flow assessment in patients with normal pregnancy revealed homogenous placental tissue, normal distribution of vessels, and active blood flow (Fig.1); in patients with pregnancy complications, we found inhomogeneous placenta, decreased blood flow, sporadic vessels, and avascular areas (Fig.2).

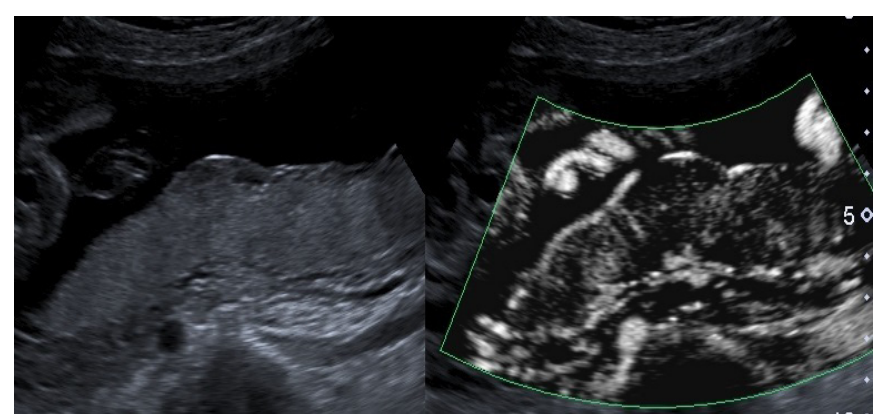

Fig. 1. Normal placenta (left-B-mode, right-SMI).

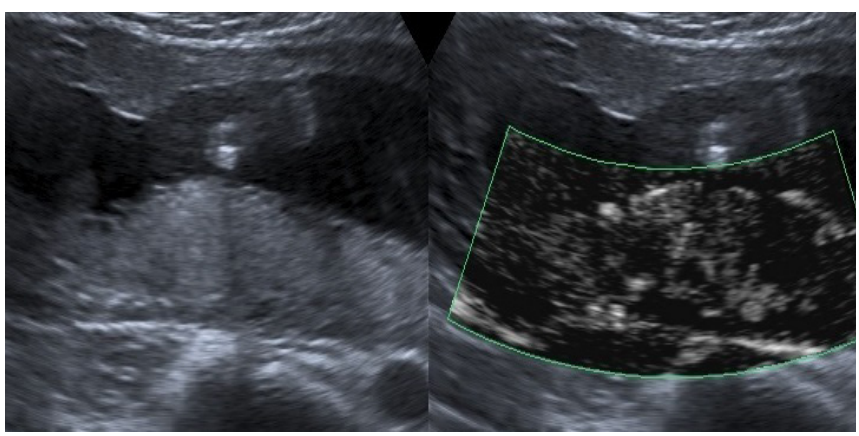

Fig 2. Pathological placenta (left-B-mode, right-SMI).

Evaluation of SMI results (Fig. 3-8) in a selected area included the pattern and distribution of vessels. The amount of spiral arteries in the area is a marker of normal/pathological 
placentation. The vascularization index (VI) was calculated automatically. VI values were from $15.2 \%$ to $44.1 \%$ for all cases; mean values varied significantly in two groups (Table 1).

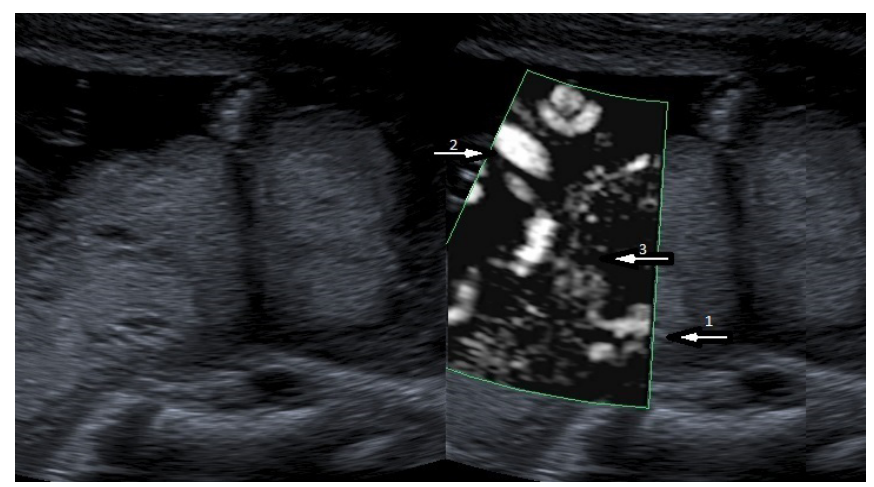

Fig 3. An area of normal placenta and umbilical cord left$B$-mode, right-SMI. 1 - spiral arteries (SAs), 2 - umbilical cord, 3 - microvessels).

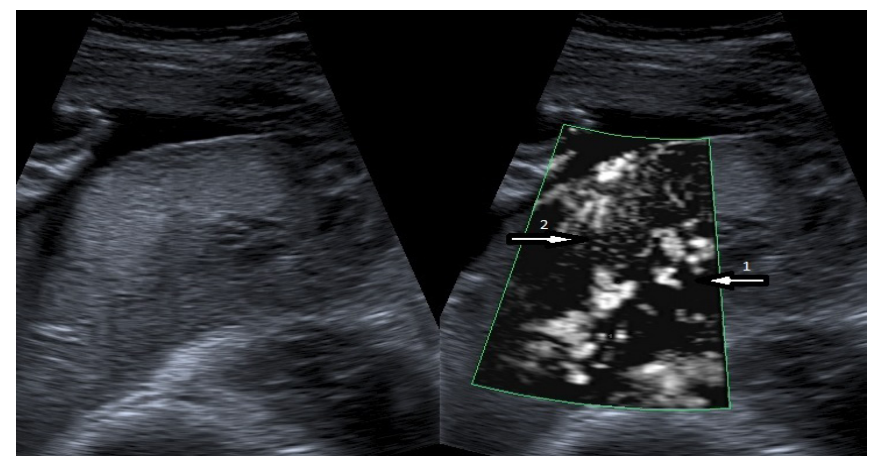

Fig 4. An area of normal placenta (left-B-mode, right-SMI. 1 -SAs, 2 - microvessels).

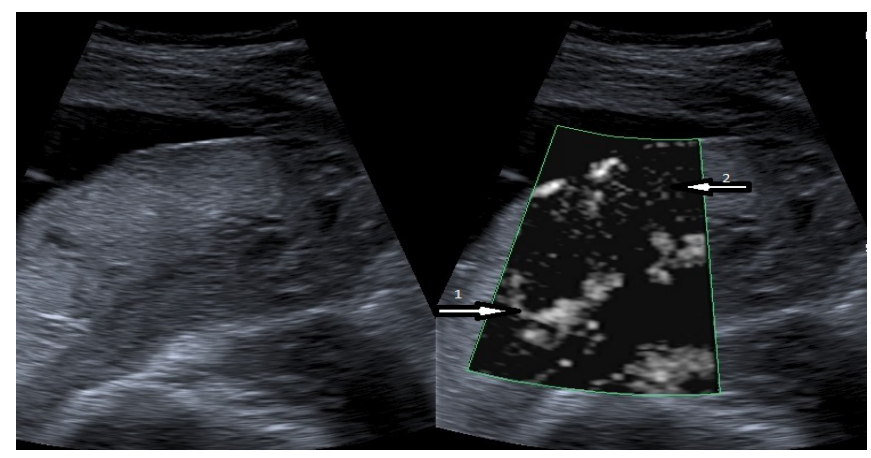

Fig 5. An area of normal placenta (left - B-mode, right-SMI. 1-SAs, 2 - microvessels).

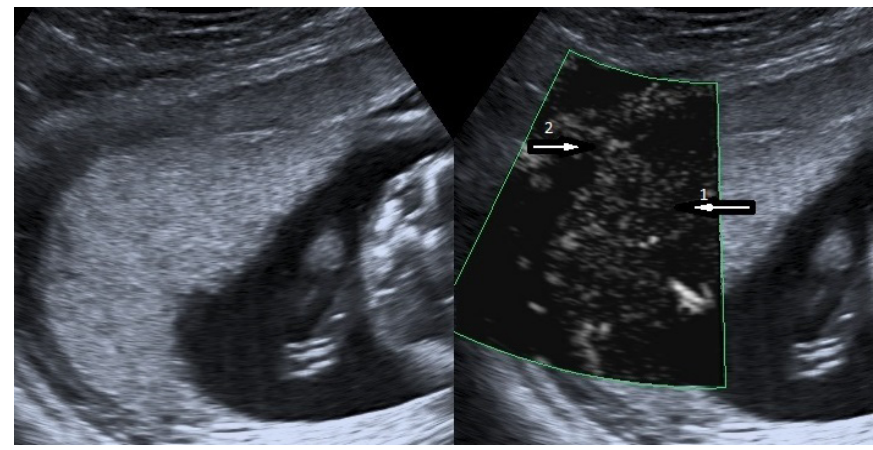

Fig. 6. An area of placenta in patient with threatened miscarriage (left-B-mode, right-SMI. 1-SAs, 2 - microvessels).

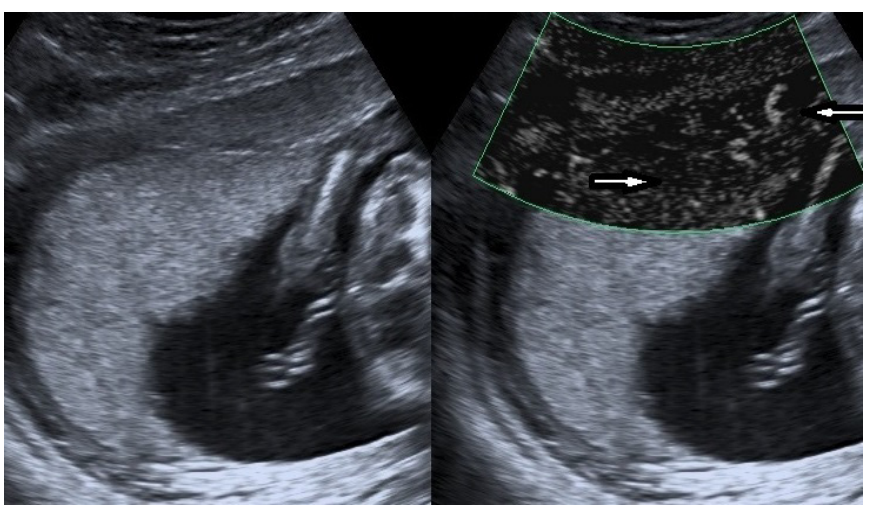

Fig 7. An area of placenta in patient with threatened miscarriage (left-B-mode, right-SMI. 1 -SAs, 2 - microvessels).

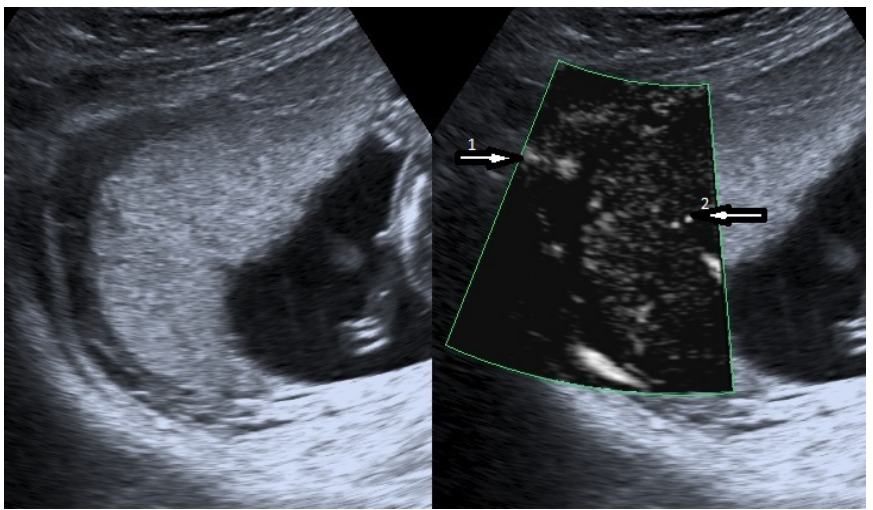

Fig 8 . An area of placenta in patient with threatened miscarriage (left-B-mode, right-SMI. 1 -SAs, 2 - microvessels).

Table 1.

Vascularization index in control and threatened miscarriage groups

\begin{tabular}{|l|c|c|c|}
\hline \multicolumn{1}{|c|}{ Parameter } & Group 1 & Group 2 & P \\
\hline $\begin{array}{l}\text { VI, \% } \\
\text { Mean (range) }\end{array}$ & $33.6(16.1-44.1)$ & $20.3(15.2-24.2)$ & $<0.05$ \\
\hline
\end{tabular}

Placental blood flow was also assessed by SMI as well as by color Doppler in the same areas of the placenta. SMI demonstrated several benefits compared to color Doppler imaging (Table 2).

Table 2.

Benefits of SMI compared to color Doppler imaging

\begin{tabular}{|l|c|c|}
\hline \multicolumn{1}{|c|}{ Criteria } & SMI & $\begin{array}{c}\text { Color Doppler } \\
\text { imaging }\end{array}$ \\
\hline Maximum spatial resolution & + & - \\
\hline $\begin{array}{l}\text { Lower resistance vessels and } \\
\text { microvessels visualization }\end{array}$ & + & - \\
\hline High frame rate and scale & + & - \\
\hline Artifacts & - & + \\
\hline
\end{tabular}


Color Doppler allows us to assess superficial vessels only, whereas SMI provides more comprehensive data on the overall vascularization of the placenta (Fig. 9-12).

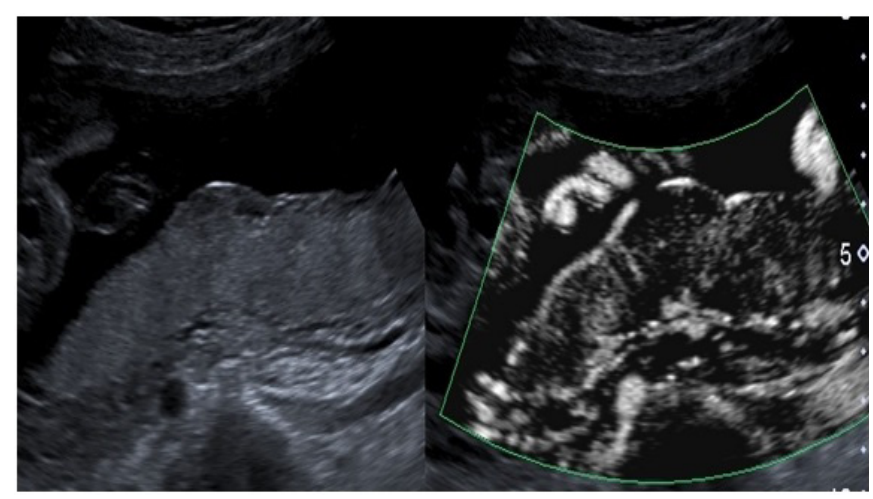

Fig. 9. Normal placenta (left-B-mode, right-SMI).

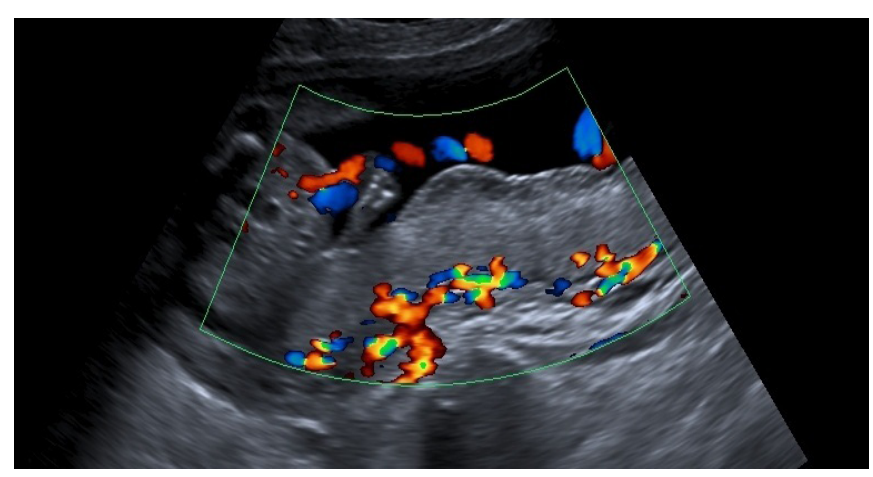

Fig. 10. Normal placenta (color Doppler imaging)

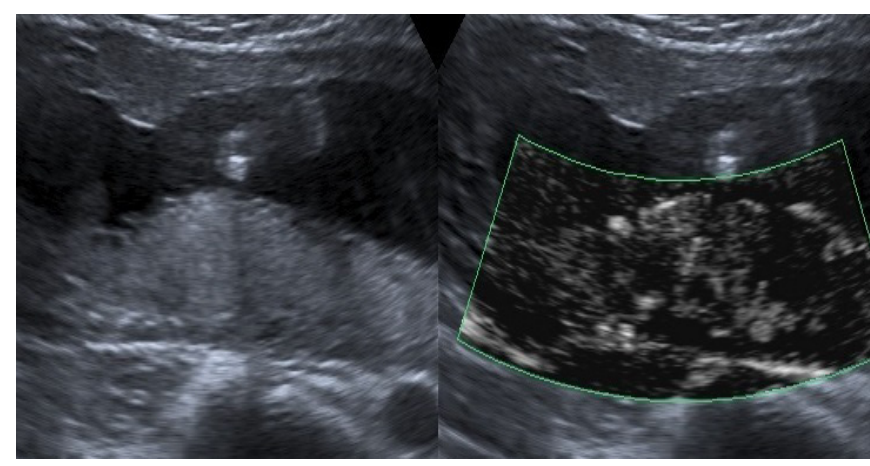

Fig. 11. Placenta in patient with threatened miscarriage (left - B-mode, right-SMI).

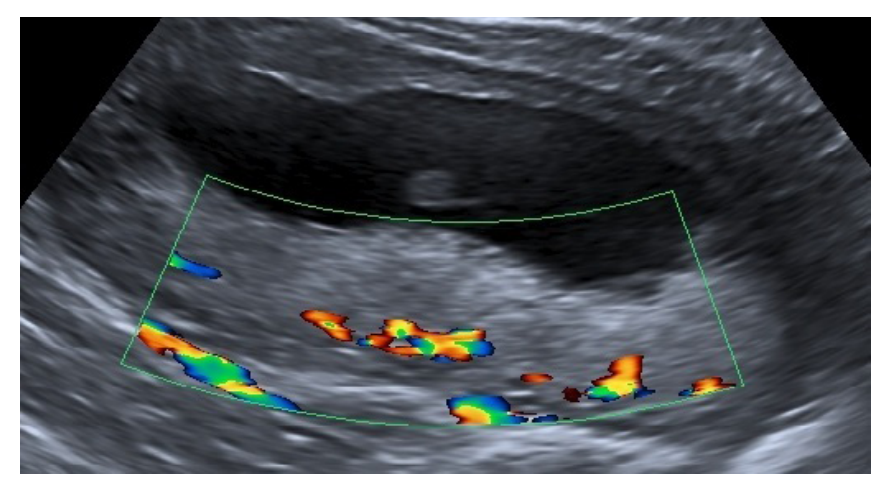

Fig. 12. Placenta in patient with threatened miscarriage(color Doppler imaging).

\section{Conclusion}

The SMI technique is beneficial in patients with complications during pregnancy due to the ability to detect microcirculatory abnormalities and vascular homomorphism, as well as abnormal transformation of spiral arteries. Those features are typical for placental insufficiency. Results of the current study demonstrate that SMI allows controlling the placental circulation. Assessment of the patterns of placental vessels may provide an opportunity for early diagnosis of blood flow alterations and early onset of treatment. Compared to color Doppler imaging SMI demonstrated several beneficial characteristics: color Doppler imaging is inappropriate for lower resistance vessels and for visualization of microvessels. This means that color Doppler does not provide enough data on placental blood flow, thus making early diagnosis of placental insufficiency impossible. SMI by Aplio ${ }^{\mathrm{TM}} 500$ (Toshiba) may be an effective tool in the assessment of placental blood flow and the diagnosis and prognosis of placental insufficiency.

\section{Competing interests}

The authors declare that they have no competing interests.

\section{References}

1. Shirokova VI, Filippiv OS, Guseva EV. Women's health and the basic directions in the development of obstetric services in the Russian Federation. Zdravookhraneniye. 2009; $11: 25-31$

2. Kuzmin VN. Fetoplacentar insufficiency: a problem of the modern obstetrics. Lechashchii Vrach. 2011;3:50-4.

3. Milovanov AP, Savelyeva SV. The intrauterine development of a human. M.: MVV; 2006.

4. Dobrokhotova YuE, Milovanov AP, Djobava EM, Dzeygova EA. The placental insufficiency in women with a chronic venous insufficiency: some aspects of the etiology, pathogenesis and diagnosis. Rossiyskiy Vestnik AkusheraGinekologa. 2007;7(1):12-8.

5. Burton GJ, Charnok-Jones DS, Jauniaux E. Regulation of vascular growth and function in the human placenta. Reproduction. 2009; 138(6):895-902. doi:10.1530/REP-09-0092.

6. Medvedev MV. Prenatal echography: differential diagnosis and prognosis. M.: The Real Time; 2012.

7. Ozerskaya IA. Echography in gynecology. M.: VIDAR; 2013.

8. Andronova NV, Zaretskaya NV, Khodjaeva ZS, Shegolev AI, Khramtchenko NV, Voevodin SM. Placental pathology in fetal chromosome abnormalities. Akush Ginekol (Mosk). 2014;3:4-8.

9. Burton GJ, Jauniaux E, Charnock-Jones DS.. The influence of the intrauterine environment on human placental development. Int J Dev Biol. 2010;54(2-3):303-12. doi: 10.1387/ijdb.082764gb. Review.

10. Titchenko LI, Pyrsikova JYu, Chechneva MA, Titchenko IP. Three-dimensional ultrasound examination for the functional assessment of intraplacentar vasculature. SonoAce Ultrasound. 2007;16. http://www.medison.ru/si/art254.htm 11. Mihu CM, Suşman S, Rus Ciucă D, Mihu D, Costin N. Aspects of placental morphogenesis and angiogenesis. Rom J Morphol Embryol. 2009;50(4):549-57. 